\title{
Imaging of Gastric Carcinoma. Part One: Diagnosis and Staging
}

\author{
Muhammad O. Awiwi ${ }^{1} \quad$ Rochita V. Ramanan ${ }^{2}$ Mohamed Elshikh ${ }^{3}$ Raghunandan Vikram \\ 1 Department of Diagnostic Imaging, The University of Texas MD \\ Anderson Cancer Center, Texas, United States \\ 2 Apollo Hospital Chennai, India \\ ${ }^{3}$ Department of Radiology, The University of Texas Medical Branch, \\ Texas, United States \\ Address for correspondence Muhammad O. Awiwi, MD, Department \\ of Diagnostic Imaging, The University of Texas MD Anderson Cancer \\ Center, Texas, United States \\ (e-mail: mohammad_owiwi@hotmail.com).
}

J Gastrointestinal Abdominal Radiol ISGAR 2021;4:194-205.
Abstract
Keywords
- Stomach
- adenocarcinoma
- computerized tomography
- radiology
- review
- staging

Gastric cancer is one of the leading causes of death from malignancy. Despite the enormous advancement in medical oncology over the past decade, surgical resection of early tumors remains the most effective treatment. Accurate interpretation of radiologic imaging studies is crucial for staging local disease spread, predicting possible lymphatic involvement, and identifying metastatic disease, thereby guiding management plans. This article reviews imaging patterns of the normal stomach along with appearances of gastric cancer, its local spread patterns and distant metastasis, and also describes key features pertaining to preoperative staging.

\section{Introduction}

Less than a century ago, gastric carcinoma was the most common cancer in the world. Since then, the incidence and mortality rates of gastric carcinoma have been decreasing. It is currently the fifth most common cancer worldwide and accounts for $8.2 \%$ of all cancer-related deaths globally. ${ }^{1}$ There is substantial geographic heterogeneity in the incidence of gastric carcinoma, wherein the incidence can vary up to 10fold between high-risk and low-risk countries. There are several reasons why this difference persists such as differences in $\mathrm{H}$. pylori infection rates, cigarette smoking, and use of salt and salt-preserved foods, which are established carcinogens associated with gastric carcinoma. Estimates from the global burden of diseases, injuries, and risk factors study of 2017 suggest that Mongolia and Afghanistan had the highest age-standardized incidence rates with 37.6 and 33.6 per 100,000 population. It is notable that high age-standardized incidence rate is seen generally in the high-income Asia Pacific region (Japan, South Korea), with incidences of 29.5 per thousand population, followed by Eastern Europe and Andean Latin America. In contrast, India has relatively low rates of gastric carcinoma, with age-standardized incidence rate of 7.5 per 100,000 population. $^{2}$

Gastric carcinoma presents at an advanced stage and has a poor prognosis. Some countries with high incidence of gastric carcinoma (such as Japan) have national screening programs. These programs allow for more patients getting diagnosed with gastric carcinoma at an early stage when the disease is potentially curable. ${ }^{3}$ For localized gastric carcinoma, the best chance for durable disease control and survival is curative surgery. The overall survival for gastric carcinoma is poor and the 5 -year survival rate with surgical treatment alone ranges between 23 to $25 \%$. Hence, multimodality published online November 8, 2021
DOI https://doi.org/ 10.1055/s-0041-1735217. ISSN 2581-9933.

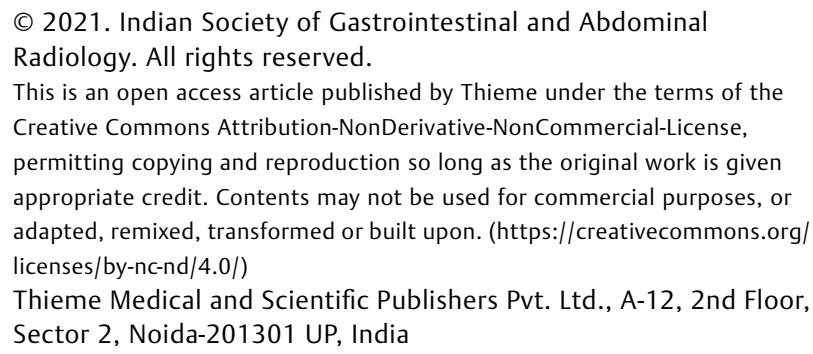


therapy with either preoperative or postoperative chemoradiotherapy is employed to improve rate of disease control and minimize disease recurrence.

\section{Clinical Presentation}

Signs and symptoms of gastric carcinoma are nonspecific. Hence, the diagnosis is often late and patients generally have advanced disease at the time of presentation. Weight loss and persistent abdominal pain are the most common symptoms at initial diagnosis. Advanced cancer of the proximal stomach is a cause of pseudoachalasia, which may present with dysphagia and mimic achalasia on upper gastrointestinal (GI) barium studies. Nausea or early satiety may result from the tumor mass occupying the gastric lumen. This symptom may also be seen in patients with diffuse gastric carcinoma (linitis plastica), due to disruption of gastric peristalsis and loss of gastric distensibility. Occult GI bleeding with or without iron deficiency anemia is seen in a few patients. Some patients present with signs or symptoms related to distant metastatic disease such as palpable left supraclavicular node (Virchow's node), periumbilical nodule (sister Mary Joseph node), and left axillary node (Irish node). Peritoneal spread can present with ascites and abdominal distention. Jaundice or clinical evidence of liver failure can be seen in advanced metastatic disease involving the liver. Rarely, patients with advanced gastric cancer may involve the adjacent transverse colon, causing feculent emesis or severe diarrhea related to gastrocolic fistula. Nausea, vomiting, and abdominal distention can occur due to large bowel obstruction or gastric outlet obstruction (GOO). Gastric adenocarcinoma is also associated with deep venous thrombosis, which when treated with anticoagulants may cause upper GI bleed from the gastric mass.

\section{Anatomy}

The stomach is a derivative of the foregut, and it is suspended at its location by five ligaments: the gastrohepatic ligament, the hepatoduodenal ligament, the gastrosplenic ligament, the splenorenal ligament, and the gastrocolic ligament. The gastrohepatic and hepatoduodenal ligaments together form the lesser omentum, and they extend between the liver hilum and the lesser curvature of the stomach and the proximal duodenum. The gastrocolic ligament extends between the greater curvature of the stomach and the transverse colon, and it forms the greater omentum. The gastrosplenic ligament extends between the hilum of the spleen and the gastric fundus along with the greater curvature of the stomach. The splenorenal ligament is a misnomer, and this ligament extends between the hilum of the spleen and the anterior pararenal space, which contains the pancreatic tail and splenic vessels. ${ }^{4,5}$

The gastric ligaments are covered by peritoneal lining, and they contain adipose tissue, nerves, lymphatics and blood vessels. On CT, blood vessels that coarse through these ligaments serve as landmarks to identify these ligaments. The arcade of the left and right gastric arteries courses within the gastrohepatic ligament. The portal triad (hepatic artery, portal vein, and common bile duct) passes through the hepatoduodenal ligament. The arcade of the right and left gastroepiploic arteries courses within the gastrocolic ligament. The gastrosplenic ligament is close to the splenic hilum, and it contains the short gastric and left gastroepiploic arteries. The splenorenal ligament contains the distal splenic artery and vein. ${ }^{5}$

Gastric cancer can spread though lymphatics contained within the gastric ligaments, ultimately draining into the para-aortic lymph nodes. Alternatively, a tumor breaching the subserosal connective tissue of the stomach can directly spread through the adipose tissue of gastric ligaments without perforating its peritoneal lining, which is termed subperitoneal dissemination. On cross-sectional imaging, subperitoneal dissemination appears as sheet-like thickening or soft-tissue nodularity along the gastric ligaments. ${ }^{5}$

\section{Classification}

There are several classification systems proposed to describe gastric carcinoma. Clinically, an important differentiation is the differentiation between cancers involving the gastric cardia (gastroesophageal junction) and cancers involving the noncardia (more distal) portions of the stomach. Rates of noncardia gastric cancer have been steadily declining over the last several decades in most populations and are likely affected by extrinsic factors such as $\mathrm{H}$. pylori infection and food preservatives. Cancers of the gastric cardia, on the other hand, have epidemiological characteristics similar to esophageal adenocarcinoma like obesity and gastroesophageal reflux disease. Cancers of the cardia are relatively more common in the West, whereas cancers of the distal stomach are more common in high risk geographic regions. At MD Anderson Cancer Center, $41 \%$ of all upper GI cancers involve the gastroesophageal junction. ${ }^{6}$

Another important classification system which has stood the test of time is the Lauren classification, which allows a better understanding of the pathology and pathophysiology of this disease. The Lauren classification differentiates gastric carcinoma, based on microscopic growth pattern into intestinal and diffuse types. ${ }^{7}$ Diffuse cancers exhibit deep infiltration of the gastric wall and show little or no gland formation, and they are associated with inflammation and desmoplasia with relative sparing of the mucosa. The intestinal type shows glandular proliferation, similar to that seen in the large intestine. These can be well- to poorly differentiated and tend to show an expansile growth pattern rather than an infiltrative pattern. Diffuse cancers are seen mainly in younger individuals likely secondary to genetic predisposition. ${ }^{8}$ Diffuse cancers are relatively more common in the West. Intestinal type cancer, on the other hand, is believed to be related to environmental factors. It is relatively more common in high-risk regions. The steady decline in the incidence of gastric carcinoma incidence and mortality is attributed to the decrease in the incidence of intestinal type of adenocarcinoma. 8,9

In addition to environmental factors such as diet and Helicobacter pylori infection, gastric carcinoma can occur 
as a part of hereditary nonpolyposis colon cancer syndrome and other gastrointestinal polyposis syndromes such as Peutz-Jeghers syndrome and familial adenomatous polyposis. ${ }^{10}$ Mutations in the E-cadherin gene are a well-recognized cause of hereditary diffuse gastric cancer. ${ }^{11}$

Another system of classification is based on histopathology and cell origin: epithelial, mesenchymal, neuroendocrine tumors, and lymphoma. Malignant epithelial tumors constitute most malignant tumors of the stomach, about $90 \%$ of which are adenocarcinomas. Gastric carcinomas are pathologically further subclassified, based on histological appearance into tubular, papillary, mucinous, and signet-ring cell carcinomas. Based on gross macroscopic appearance, it is classified into four types. Type I is a polypoid and fungating variety, type II is a polypoid tumor with central ulceration, type III is an ulcerated tumor with infiltrative margins, and type IV is diffuse infiltrative type or linitis plastica. This method of classification is called the Borrmann's classification and is generally reserved for advanced cancers. ${ }^{12}$ However, the most widely accepted and clinically used classification is the Lauren's classification.

\section{Staging}

The tumor, node, and metastases (TNM) staging system is the most widely accepted method for staging gastric carcinomas, ${ }^{13}$ and it is depicted on - Table 1. - Table 2 summarizes key radiologic imaging features that need to be described for staging of gastric malignancies. Depth of tumor invasion is an important determinant of overall survival. For example, T1 cancers have a 10 -year survival rate of about $80 \%$, which drops to $55 \%$ in T2 cancers. T3 and T4 cancers have a 10 -year survival of only $30 \%{ }^{14-17}$

The Japanese research society for gastric carcinoma has classified regional lymph nodes, based on location into four compartments 1 to $4 .^{18}$ Compartment 1 consists of perigastric lymph nodes immediately adjacent to the stomach along unnamed arteries. Compartment 2 are nodes along the left gastric artery, the common hepatic artery, the celiac axis, and the splenic artery. Compartment 3 are nodes along the hepatoduodenal ligament, nodes posterior to the head of the pancreas, and nodes at the root of the small bowel mesentery. Compartment 4 includes para-aortic lymph nodes. The extent of lymph node dissection in surgery is described, based on surgical dissections of these compartments. D1 dissection includes compartment 1, D2 dissection includes compartments 1 and 2, D3 dissection includes compartments 1, 2 and 3, whereas D4 dissection includes dissection of all four compartments. Surgical practices vary widely and are mainly based on the extent of lymph node dissection. The knowledge of nodal anatomy in gastric carcinoma in an attempt to stage these accurately is one of the biggest contributions radiologists can make to triage gastric carcinoma patients for appropriate treatment.

The international committee on gastric carcinoma recommends that for accurate staging and prediction of prognosis, a minimum of 16 lymph nodes have to be included in the surgical specimen. ${ }^{19}$ The $\mathrm{N}$ stage is an independent
Table 1 The AJCC TNM classification ${ }^{13}$

\begin{tabular}{|l|}
\hline Primary tumor (T) \\
\hline Tx: Primary tumor cannot be assessed \\
\hline T0: No evidence of primary tumor \\
\hline $\begin{array}{l}\text { Tis: Carcinoma in situ: intraepithelial tumor without } \\
\text { invasion of the lamina propria }\end{array}$ \\
\hline T1: Tumor invades the lamina propria or submucosa \\
\hline T2: Tumor invades the muscularis propria or the subserosa \\
\hline T2a: Tumor invades muscularis propria \\
\hline T2b: Tumor invades subserosa \\
\hline $\begin{array}{l}\text { T3: Tumor penetrates the serosa (visceral peritoneum) } \\
\text { without invading adjacent structures }\end{array}$ \\
\hline T4: Tumor invading adjacent structures \\
\hline Regional lymph nodes (N) \\
\hline Nx: Regional lymph node(s) cannot be assessed \\
\hline N0: No regional lymph node metastasis \\
\hline N1: Metastasis in one to six regional lymph nodes \\
\hline N2: Metastasis in 7 to 15 regional lymph nodes \\
\hline N3: Metastasis in more than 15 regional lymph nodes \\
\hline Distant metastasis (M) \\
\hline Mx: Distant metastasis cannot be assessed \\
\hline M0: No distant metastasis \\
\hline M1: Distant metastasis \\
\hline
\end{tabular}

Abbreviations: AJCC, American Joint Committee on Cancer; TNM, tumor, node, and metastases.

Table 2 Key imaging findings that need to be included while staging gastric carcinoma

\begin{tabular}{|l|}
\hline Imaging radiology report keys \\
\hline Primary tumor (T) \\
\hline o Lesion morphology: polypoid, ulcerated or diffuse \\
o Location: cardia, fundus, body, antrum, pylorus, \\
greater curvature, lesser curvature. \\
o Local extent: presence of any extraluminal extension into \\
the adjacent ligaments, direct invasion of nearby organs. \\
\hline Lymph node (N) \\
o Presence, size location and number of lymph nodes in \\
the expected nodal basins draining the site of the \\
primary tumor. \\
\hline Metastases (M) \\
o Liver metastases. \\
o Peritoneal deposits (ascites is suggestive for peritoneal \\
involvement). \\
o Special attention to nodes in the posterior to the head \\
of pancreas, in the para-aortic region and in the \\
supraclavicular region. These are considered distant \\
metastases.
\end{tabular}

variable for survival. N0 disease has a 10-year survival of $70 \%, \mathrm{~N} 1$ has a survival of $41 \%$, and N2/N3 have a 10 -year survival rate of less than $30 \%{ }^{20}$ Nodal involvement is 
discussed in more detail in this issue in the article "Lymph node mapping in gastric carcinoma."

\section{Imaging Evaluation}

Radiologic and endoscopic ultrasound staging are important in diagnosis and management of gastric carcinoma patients. Various imaging techniques including double-contrast upper gastrointestinal (UGI) barium examinations, multidetector CT (MDCT), MRI, positron emission tomography-CT (PET-CT), and endoscopic ultrasound (EUS) are all used in the diagnosis and staging of gastric carcinoma to varying degrees.

Optimal gastric distention is essential for imaging of stomach malignancy, and it may be achieved through administration of effervescent granules or by consumption of $800 \mathrm{~mL}$ of water as a negative contrast agent. Dynamic multiphasic CT scanning is performed after injection of approximately $85 \mathrm{~mL}$ of intravenous (IV) contrast at a rate of 2.5 to $3.0 \mathrm{~mL} / \mathrm{second}$. Arterial phase imaging is performed at 25 to 40 seconds after starting the injection of IV contrast, the venous phase at 60 to 80 seconds, and delayed phase at 3 to 4 minutes. ${ }^{5,21}$ Despite the rapidly expanding role of MRI in cancer imaging, current guidelines do not recommend this modality for local staging of gastric cancer. ${ }^{22}$ However, MRI is a useful tool to characterize indeterminate liver lesions suspicious for metastasis.

Double-contrast UGI examination can be used as a screening tool for gastric cancer. It requires 6 hours of fasting, and it involves distending the gastric lumen using effervescent granules and coating its mucosal lining with high-density $(210-250 \% \mathrm{wt} / \mathrm{vol})$ barium suspension. Both functional and anatomic information of the esophagus, stomach, and duodenum can be obtained from these studies. However, endoscopy is necessary for tissue sampling and differentiating benign from malignant etiologies if an abnormality is noted on UGI series. ${ }^{5}$

\section{Tumor Detection and Staging}

Screening programs for gastric cancer have been implemented in high-income east Asian countries where there is a higher incidence of gastric cancer. Both endoscopy and double contrast UGI barium studies were proven to be effective for gastric cancer screening, and Japanese guidelines recommend these modalities for screening adults $>50$ years of age. However, the utilization of double contrast UGI barium studies has been declining. ${ }^{23}$ Currently, endoscopy is the modality of choice to detect and diagnose gastric carcinoma due to its high specificity, ability to diagnose the disease at an early stage, and the added advantage of obtaining tissue samples. In addition, it provides access to perform EUS, which offers a reliable way to T-stage the tumor at the time of diagnosis. ${ }^{24}$

Endoscopic ultrasound is superior to all other modalities in visualizing different layers of the gastric wall and perhaps is the most accurate preoperative staging modality of gastric carcinoma with an accuracy ranging between 78 to $94 \%{ }^{25,26}$ The gastric wall is seen as layers of alternating hyper and hypoechoic bands: superficial mucosa, deep mucosa, sub-

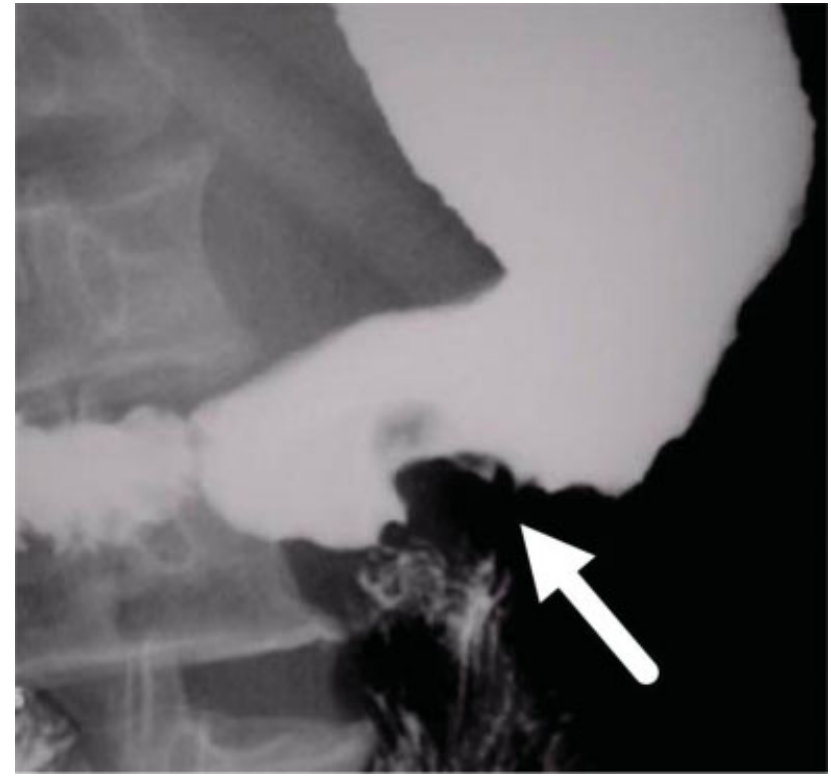

Fig. 1 Upper gastrointestinal barium examination showing an irregular polypoid mass protruding into the gastric lumen along the greater curvature of the stomach (arrow). Image courtesy: Dr Rajesh Gothi.

mucosa, muscularis propria, and serosa. Tumors are hypoechoic or hyperechoic, and they disrupt this regular wall pattern, thereby allowing for staging. ${ }^{27}$

Gastric cancer has various imaging appearances on UGI barium examination, depending on tumor morphology and on its location within the stomach. Disruption of the normal mucosal pattern, known as the areae gastricae, can be a subtle sign for the presence of a gastric mucosal lesion. Filling defects along gastric walls suggest a polypoid lesion pro-

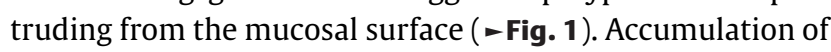
barium within an ulcer niche along with a radiolucent rim surrounding the niche is termed bull's eye appearance, and it is suggestive of an ulcer. Radiolucent rim surrounding the ulcer niche and smooth gastric folds that extend to the periphery of the ulcer are findings suggestive of a benign etiology. Findings suspicious for a malignant ulcer include an eccentric/irregular ulcer and mucosal folds which do not extend to the ulcer niche. Thick, irregular, or nodular mucosal folds in the vicinity of the ulcer are suspicious for submucosal infiltration of the disease and are also suspicious for malignancy. Rigidity and limited distensibility of the gastric lumen are suspicious for linitis plastica. Abnormal peristalsis and GOO are also suggestive of a malignant etiology (-Fig. 2). However, in the presence of these radiographic findings, endoscopy is still required for verification and tissue sampling. ${ }^{5}$

Use of high-resolution CT scanning along with multiplanar reconstructions have improved the ability of CT scan in the staging of gastric carcinoma; although, it remains inferior to EUS. Normal gastric wall shows a two- to threelayered structure: a markedly enhancing mucosa layer, a submucosal layer with lower attenuation, and an outer muscular layer invested with serosa, showing moderate 


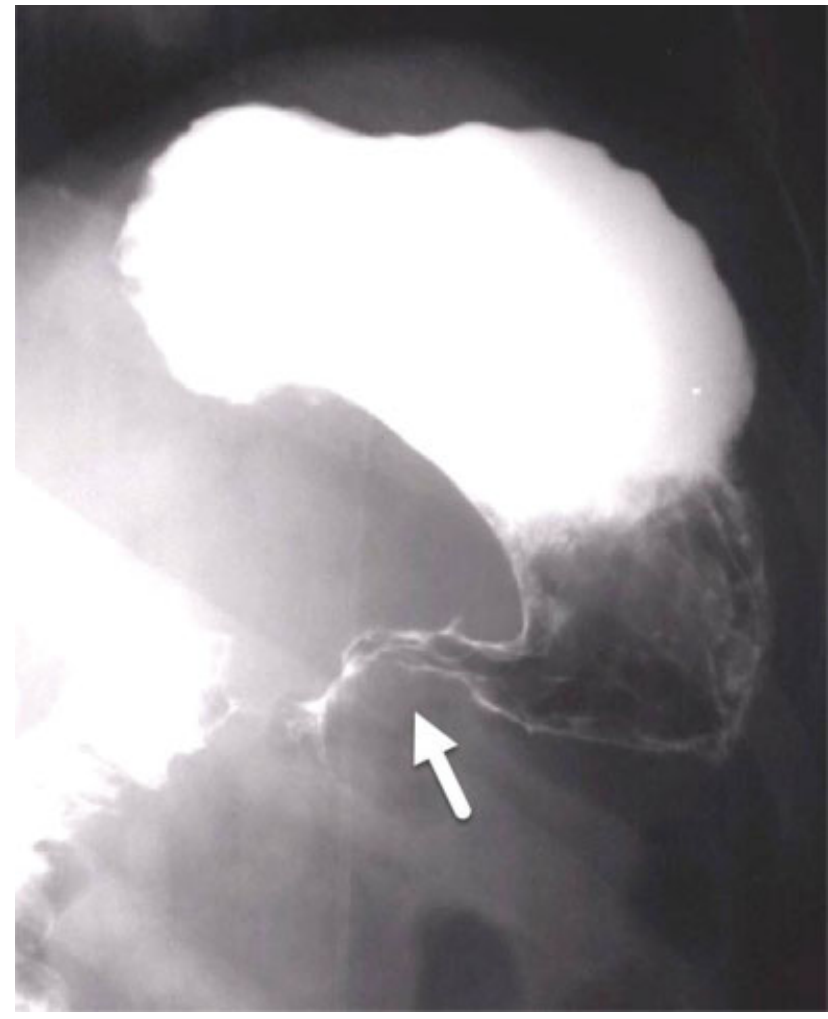

Fig. 2 Upper gastrointestinal barium examination narrowing and lack of distensibility of the distal gastric lumen with irregular contours (arrow) suggestive of adenocarcinoma. Image courtesy: Dr Rajesh Gothi.

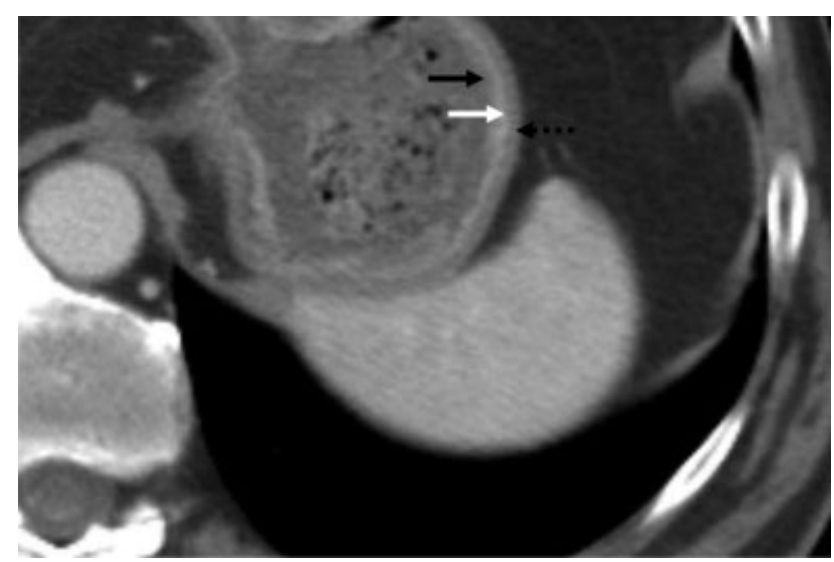

Fig. 3 Axial contrast-enhanced computed tomography of the abdomen showing alternating three layers of normal stomach: the hypervascular mucosa (solid black arrow), the hypovascular submucosa (solid white arrow), and the mildly hypervascular muscularis and serosa (dotted black arrow).

enhancement in the arterial and early portal venous phase of contrast enhanced CT (-Fig. 3$)^{28}$

Macroscopically, gastric adenocarcinoma can be classified as superficial, mass forming (-Fig. 4), ulcerative (-Fig. 5), diffuse infiltrative (-Fig. $\mathbf{6}$ ) or unclassifiable. ${ }^{29}$ Superficial tumors may be undetectable on cross-sectional imaging if they are flat, but they may be visualized when they have a

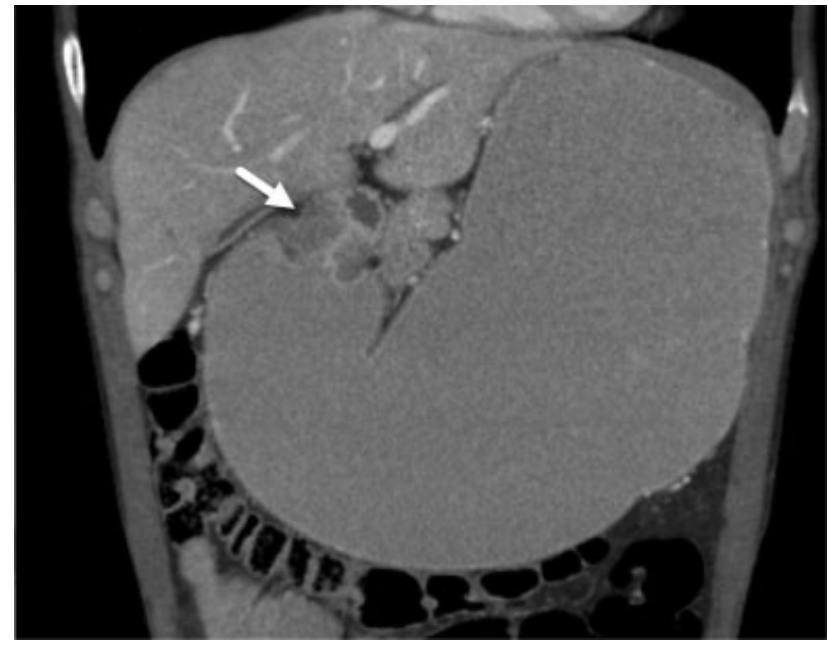

Fig. 4 An annular pyloric mass forming adenocarcinoma (arrow) causing gastric outlet obstruction with upstream dilatation of the gastric body.

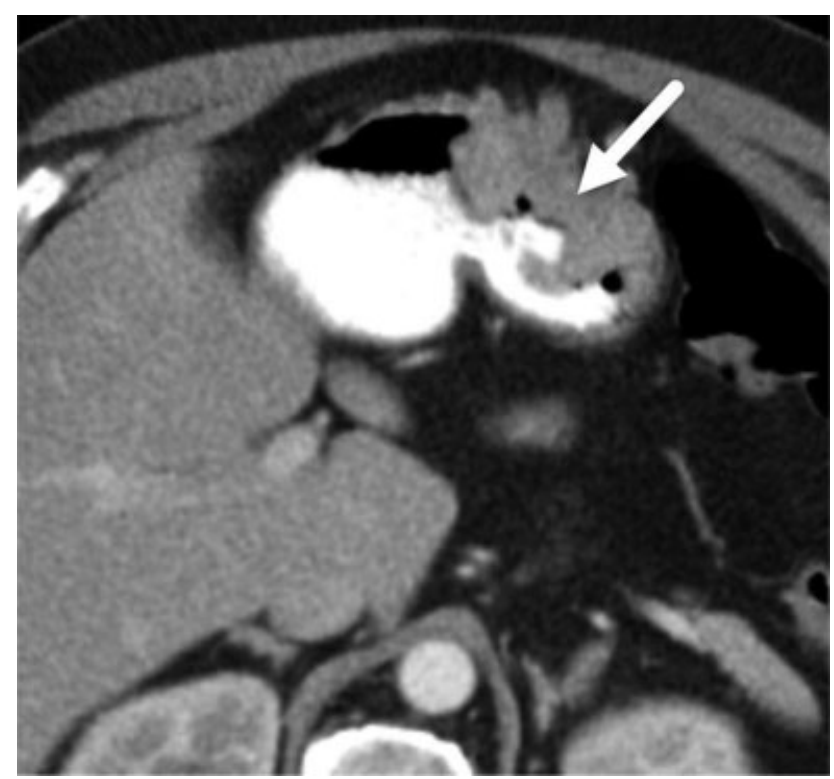

Fig. 5 A malignant ulcer along the greater curvature of the stomach (arrow) with the ulcer crater filled with oral contrast.

polypoid configuration. T1 and T2 lesions are limited to the gastric wall (-Fig. 7). T3 lesions may show slight blurring of the outer contour with stranding of perigastric fat ( - Figs. 8, 9). T4 lesions are seen spreading via ligamentous attachments and peritoneal reflections or directly invading adjacent structures. ${ }^{30}$

CT scans perform better at T-staging of advanced (T3 and $\mathrm{T} 4$ ) carcinomas ( $98 \%$ and $82 \%$, respectively) versus early (T1 and T2) carcinomas (23 and 15\%, respectively). ${ }^{21}$ Multiplanar reconstructions in coronal and sagittal planes slightly improve the detection and staging. While MRI has an advantage of multiplanar capability and better tissue contrast, the 


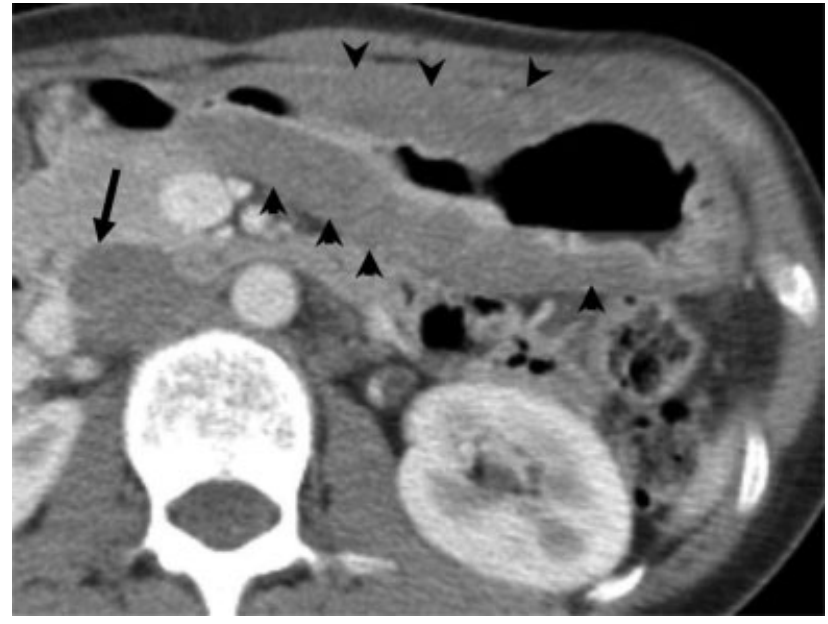

Fig. 6 Diffuse thickening involving the submucosa and muscularis layers of the stomach (black arrowheads) compatible with diffuse infiltrative gastric adenocarcinoma and linitis plastica. There is also an enlarged retroperitoneal lymph node (black arrow). The retroperitoneal node is considered a compartment 4 node in the Japanese surgical society classification and is included in D4 lymph node dissection, but it is classified as M1 disease according to the American Joint Committee on Cancer (AJCC) classification.

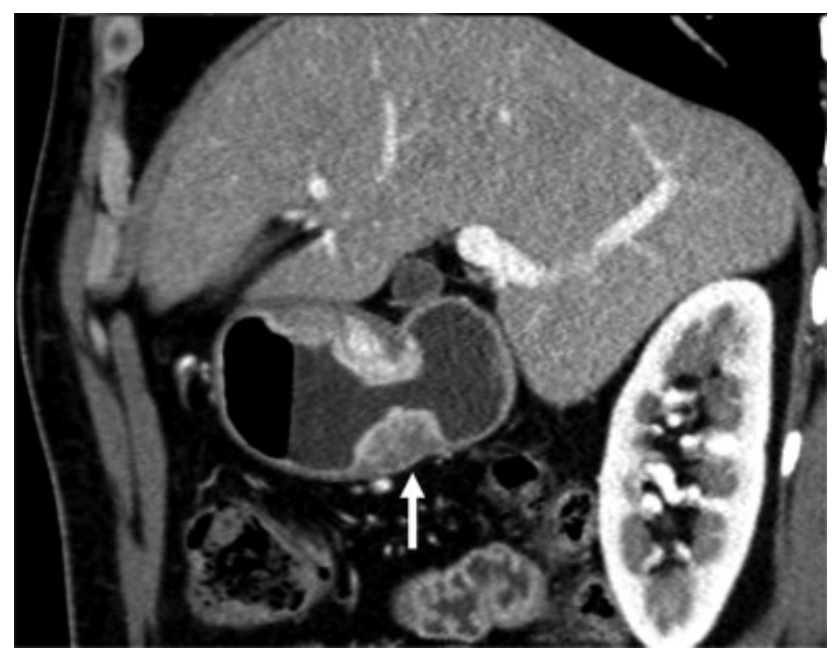

Fig. 7 Sagittal reconstruction of contrast-enhanced computed tomography scan showing a large annular mass in the gastric body. The tumor is limited to the gastric wall without evidence of blurring of the outer contour or stranding of the perigastric fat (arrow).

use of MRI in staging of the primary tumor is limited due to respiratory, cardiac, and peristalsis motion artefacts.

Fluorodeoxyglucose (FDG) PET-CT performs inconsistently in the detection of gastric carcinoma, as there is physiologic uptake of FDG in the gastric mucosa. In addition, FDG uptake varies, according to the histologic subtype of gastric cancer. Signet ring cell carcinoma and poorly differentiated adenocarcinoma show little or no radiotracer uptake. ${ }^{31,32}$

\section{Lymph Node Staging}

Nodal staging in gastric carcinoma is challenging due to significant limitations in detecting microscopic metastases

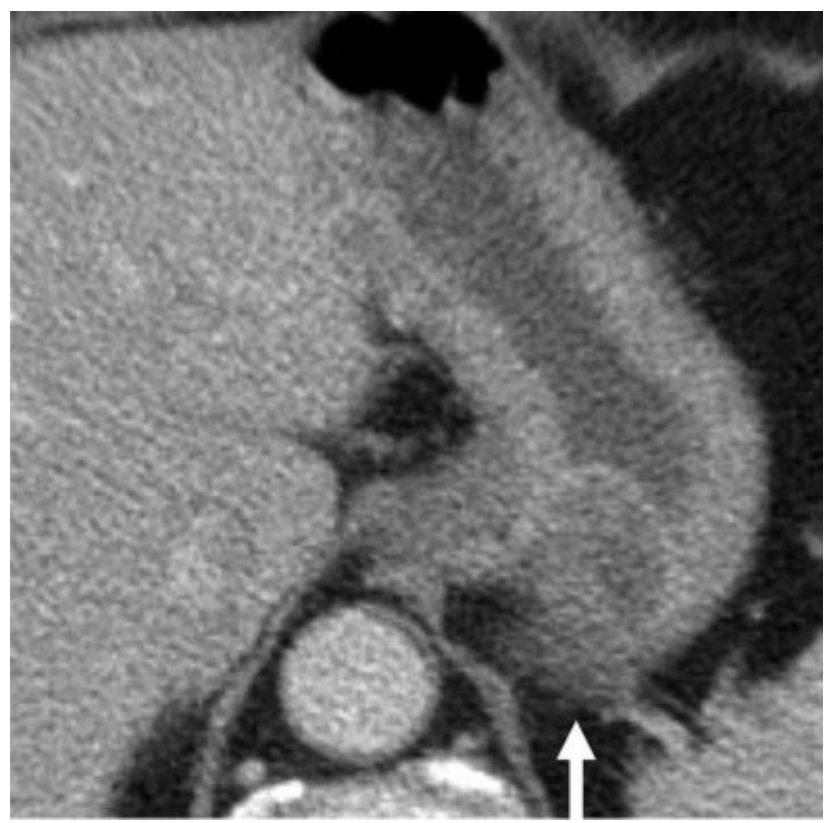

Fig. 8 Axial contrast-enhanced computed tomography scan of a patient with a tumor in the gastric fundus. There is blurring of the outer contour with stranding of the perigastric fat (arrow), indicating a T3 lesion.

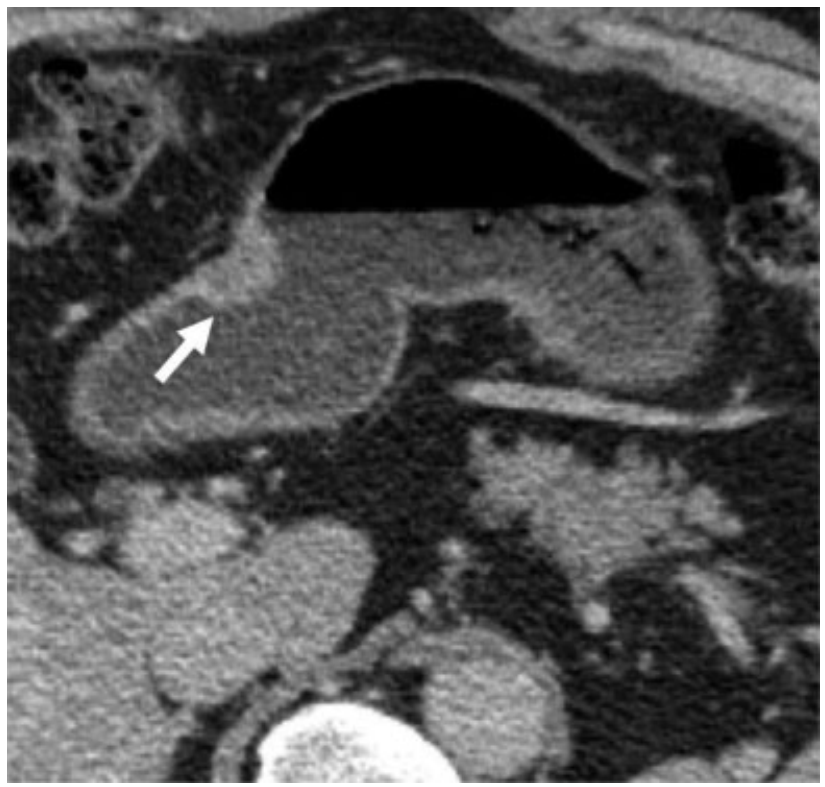

Fig. 9 Axial contrast-enhanced computed tomography scan of the abdomen in a patient with gastric adenocarcinoma, which is seen as a focal area of mucosal thickening and adjacent fat stranding. At resection, this was found to be a T3 disease (Lauren intestinal variety).

by currently available imaging modalities. In practice, nodes which are larger than 8 to 10 millimeters in short axis are considered as suspicious. However, it is humbling to note that while $80 \%$ of the lymph nodes, which were less than 5 millimeters in size, are benign, $55 \%$ of metastatic nodes are also less than 5 millimeter in short axis, clearly demonstrating the weakness of size criteria as a parameter on imaging 


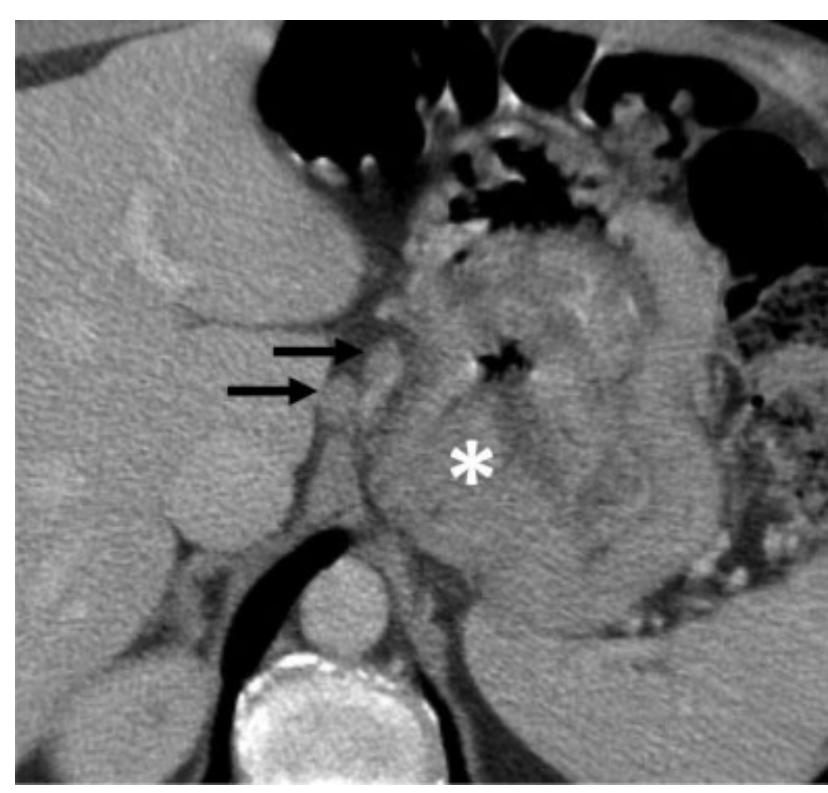

Fig. 10 Metastatic left gastric lymph nodes (black arrows) seen in a patient with advanced and diffuse gastric adenocarcinoma (asterisk). This belongs to compartment two in the Japanese research society for gastric carcinoma classification.

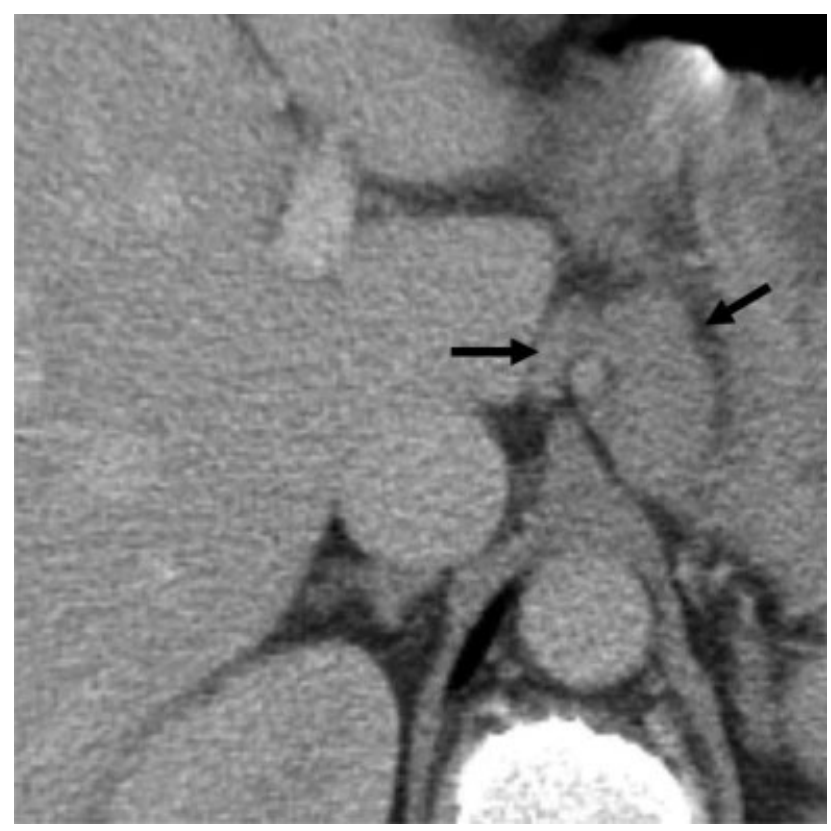

Fig. 11 Metastatic celiac artery lymph node (black arrows) in a patient with gastric adenocarcinoma.

modalities. ${ }^{33,34}$ The problems of CT scan are compounded on MRI, as it is subject to significant motion artefacts from respiration, cardiac activity, and peristalsis. Although imperfect, CT is marginally better at staging lymph nodes compared to MRI ${ }^{34-36}$ EUS can detect metastatic nodes in the perigastric region. However, important nodal stations, which are key to surgical planning (such as station 3, station 4 nodes), cannot be staged using this modality (-Figs. 6, 10-13). FDG avid nodes on PET-CT are likely malignant and

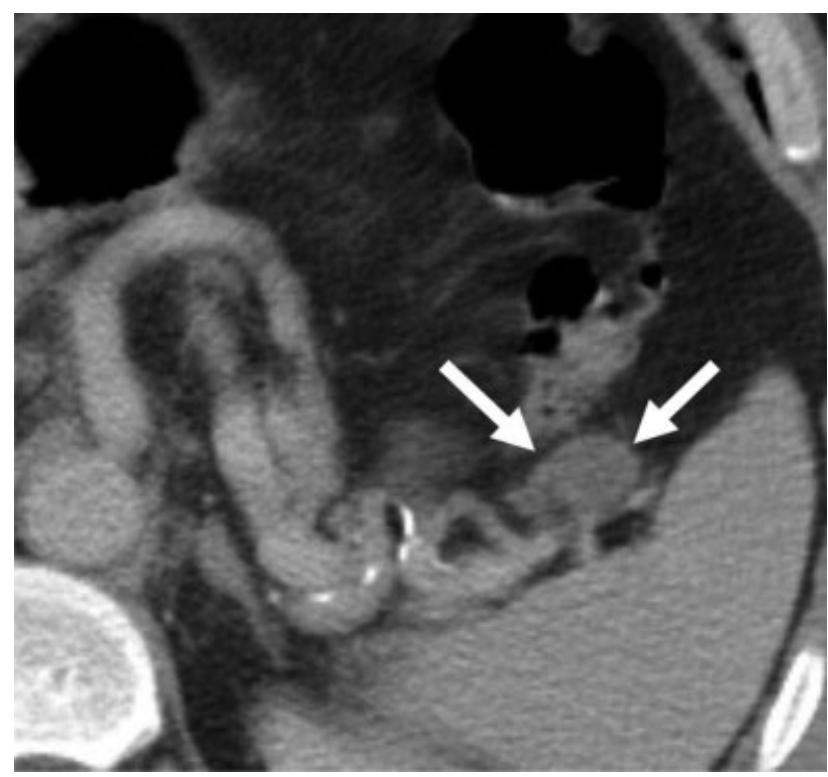

Fig. 12 Metastatic lymph node at the splenic hilum (white arrows) in a patient with gastric adenocarcinoma. This is considered as compartment 2 lymph node, according to the Japanese research society for gastric carcinoma classification. In patients with tumors of the distal stomach, the metastatic lymph node at the splenic hilum is considered M1 disease in the American Joint Committee on Cancer (AJCC) classification.

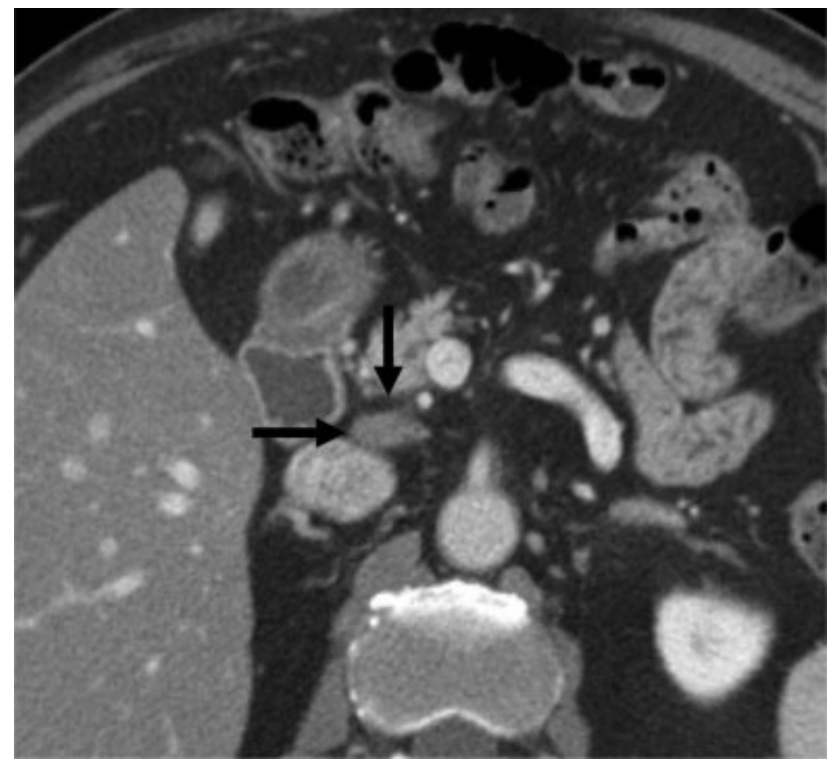

Fig. 13 Axial contrast-enhanced computed tomography scan of the abdomen shows an enlarged suspicious lymph node posterior to the pancreatic head (black arrows). This is classified as compartment III, according to the Japanese research society for gastric carcinoma, and it is considered as M1 disease according to American Joint Committee on Cancer (AJCC) classification. Compartment 3 lymph nodes are included in D3 dissection.

hence PET-CT has a higher specificity than CT or MRI (-Fig. 14). However, PET-CT has a significant size limitation, and smaller nodes may not be detectable. ${ }^{37}$ In addition, 


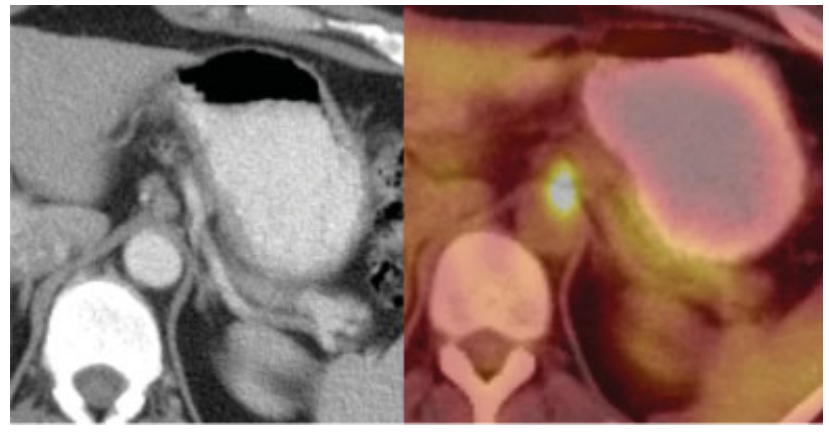

Fig. 14 Axial computed tomography (CT) scan of the abdomen and fused positron emission tomography (PET)-CT scan showing an enlarged left gastric lymph node, demonstrating avid radiotracer uptake. Elevated metabolic activity increases the specificity for predicting nodal metastasis.

signet cell carcinomas do not show FDG uptake and there is a possibility of understaging lymph nodes on PET-CT.

\section{Metastatic Gastric Carcinoma}

According to the American Joint Committee on Cancer (AJCC) staging system, cancer infiltration of the hepatoduodenal ligament ( - Fig. 15), retropancreatic, mesenteric or para-aortic lymph nodes are considered distant metastases (i.e., M1 disease). ${ }^{13}$ Hematogenous spread of gastric carcinoma is commonly seen in the liver due to its portal venous drainage, and hepatic metastatic deposits are usually hypovascular, and they are usually best seen on portal venous phase (-Fig. 16). Lung metastases are less common, and they are best detected using CT. Lung metastases appear on CT as solid lung nodules, which is a nonspecific imaging pattern (-Fig. 17). Bone metastases are more common in advanced cases. On CT bone, metastases can be sclerotic, lytic, or they may be isodense to bone marrow (-Fig. 18). The latter imaging pattern is frequently missed on CT, but it may show avid FDG uptake on PET/CT without a CT correlate. After chemotherapy, isodense can become sclerotic and hence is visible on CT. Therefore, new sclerotic bone lesions that appear after initiation of chemotherapy can be treated metastases, but new bone metastases cannot be excluded. Involvement of distant lymph nodes such as left supraclavicular node (Virchow's node) can also be seen.

Advanced gastric carcinoma frequently metastasizes to the peritoneum, typically involving the omentum, the ovaries (Krukenberg tumor) (-Fig. 19), and rectovesical pouch (Blumer's shelf tumor) (-Fig. 20). The presence of ascites in a patient with gastric carcinoma is generally associated with positive free tumor cells, which is suspicious for peritoneal carcinomatosis.

Laparoscopy is superior to CT and all other imaging modalities for staging and detection of small volume peritoneal disease. Its sensitivity for detection of peritoneal disease reaches up to $96 \%$. Some authors consider MRI to be superior to $\mathrm{CT}$ in detecting extragastric metastases. This may be particularly true for detection of hepatic metastases, but in
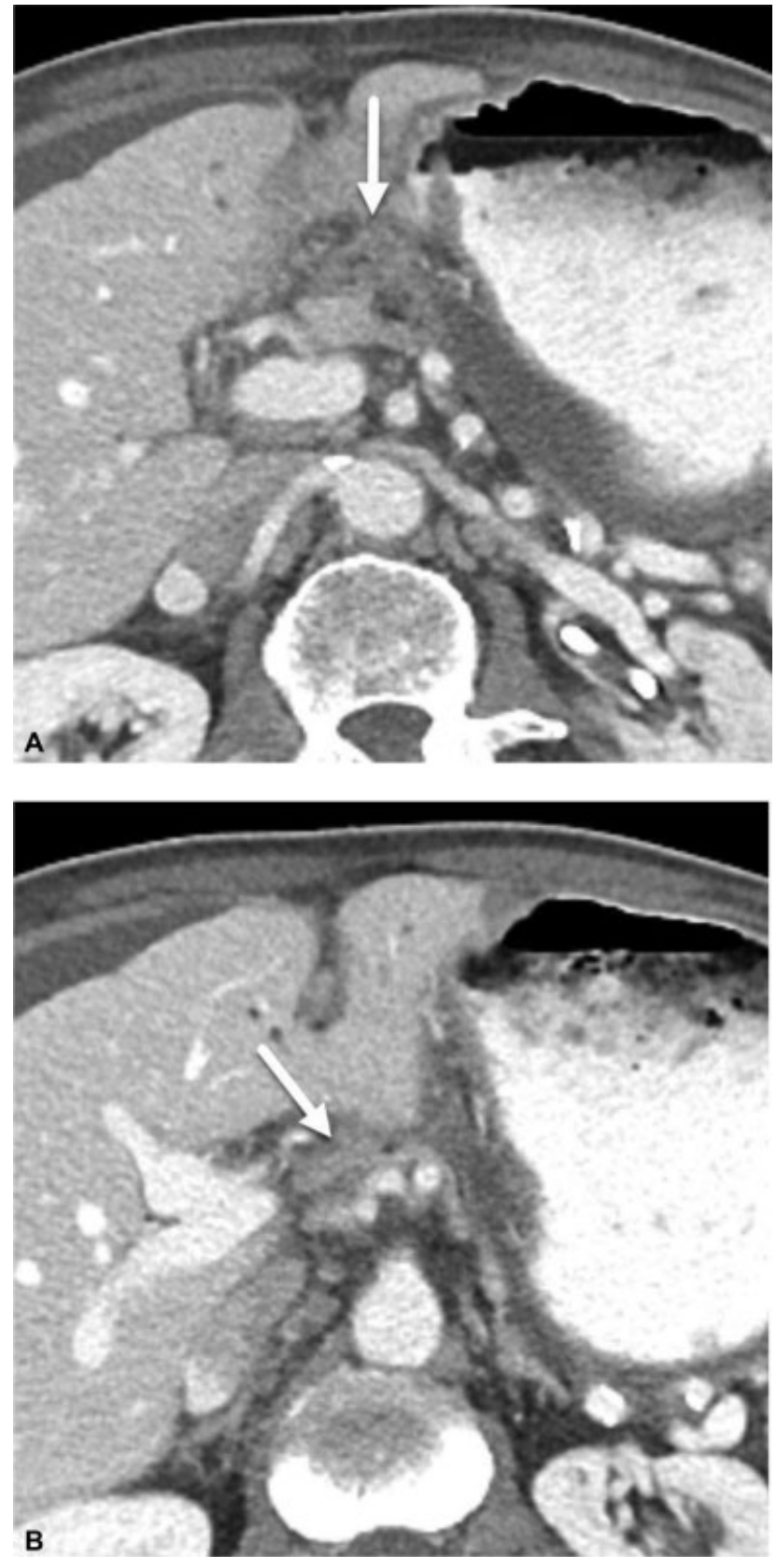

Fig. 15 Soft-tissue density lesions (arrow) along the gastrohepatic ligament $(A)$ and hepatoduodenal ligament (B) represent tumor infiltration.

the authors experience, $\mathrm{CT}$ is superior to MRI in detecting peritoneal metastases. The value of PET-CT is limited and it is still under investigation.

\section{Differential Diagnosis}

The differential diagnosis of solid gastric masses mainly includes gastric lymphoma and gastrointestinal stromal tumors (GIST). Rare causes of solid gastric tumors include metastases, leiomyoma, schwannoma, neuroendocrine tumors, lipoma, hemangioma, and glomus tumors. 


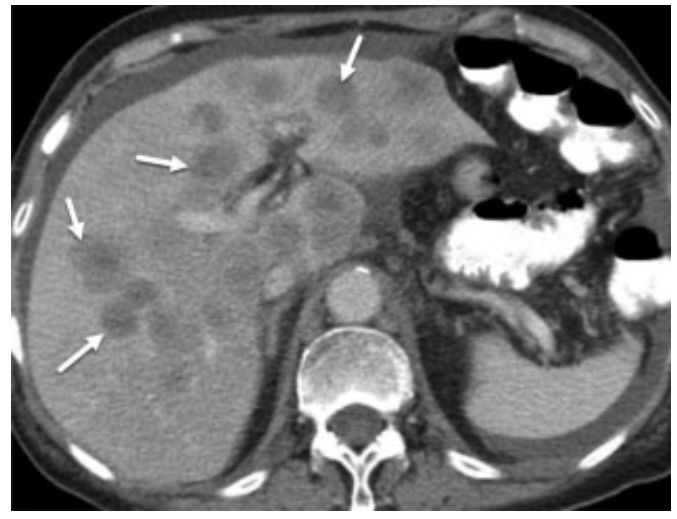

Fig. 16 Axial computed tomography scan showing multiple hepatic metastases of gastric adenocarcinoma (arrow). Adenocarcinoma metastases are typically hypoenhancing, and they are best visualized on portal venous phase.

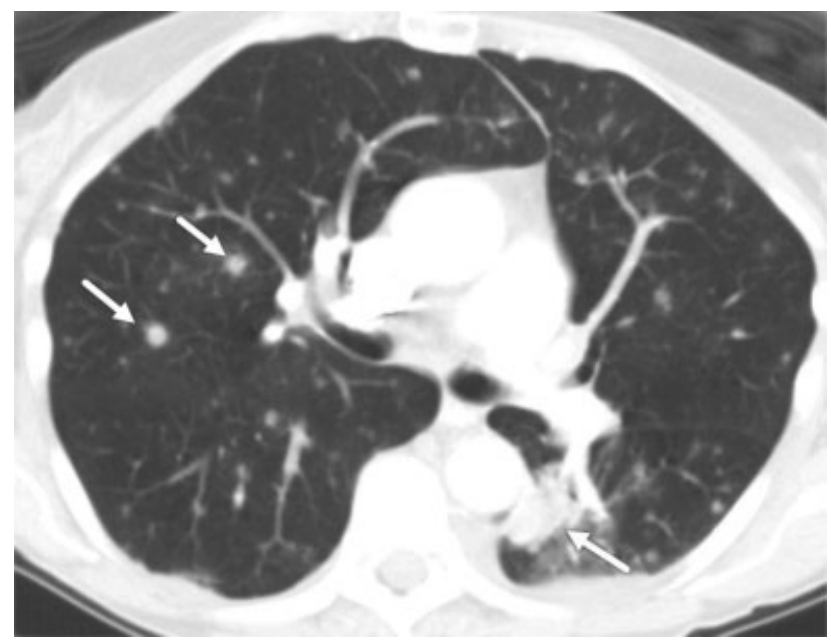

Fig. 17 Several bilateral lung nodules (arrows) suggestive of lung metastases.

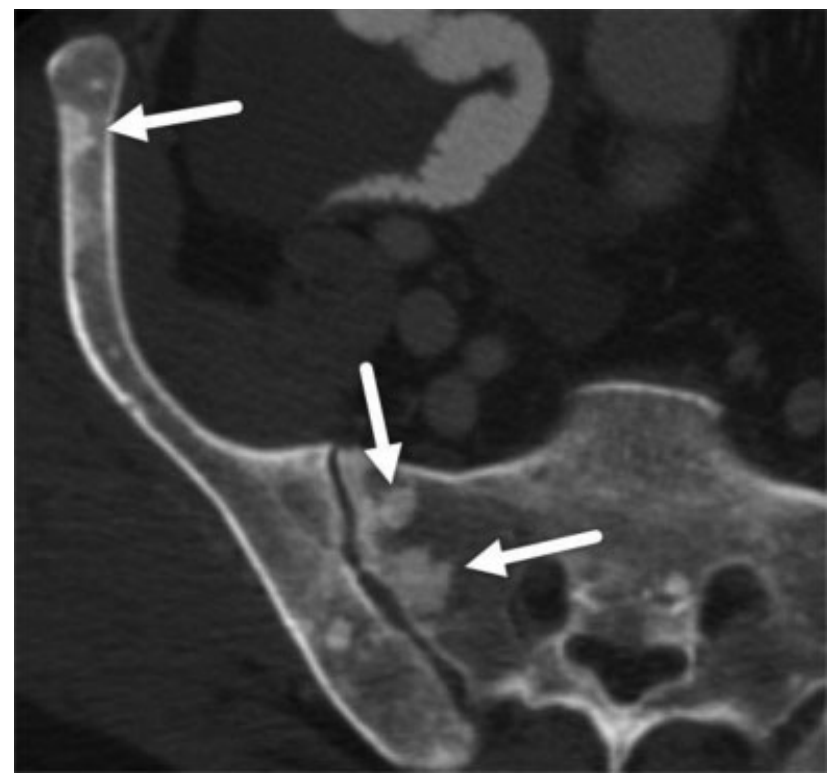

Fig. 18 Several sclerotic bone metastases (arrows) in the right sacral wing and right iliac bone.

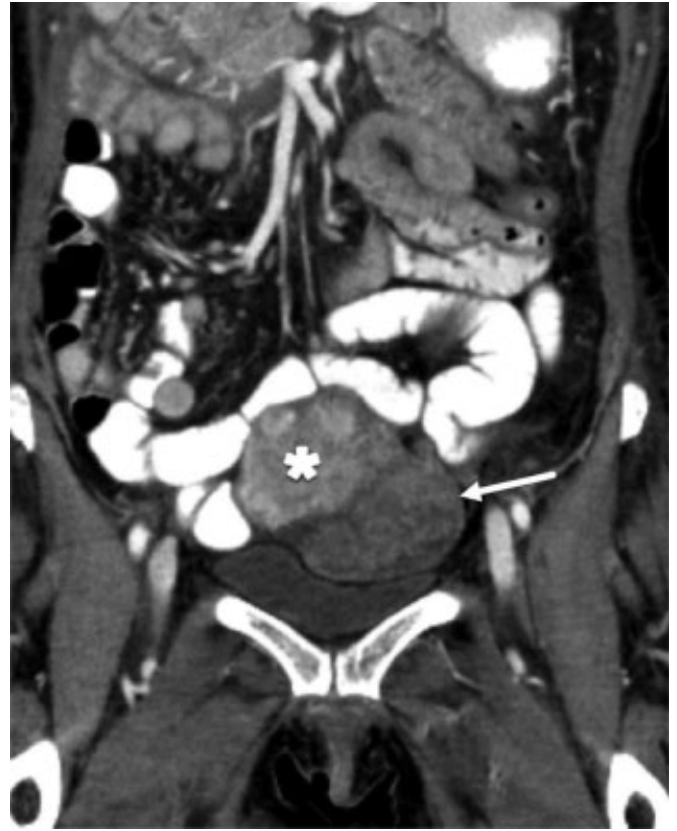

Fig. 19 A gastric adenocarcinoma patient with a left ovarian metastasis (white arrow), in keeping with Krukenberg tumor. Note the adjacent uterine fibroid (asterisk).

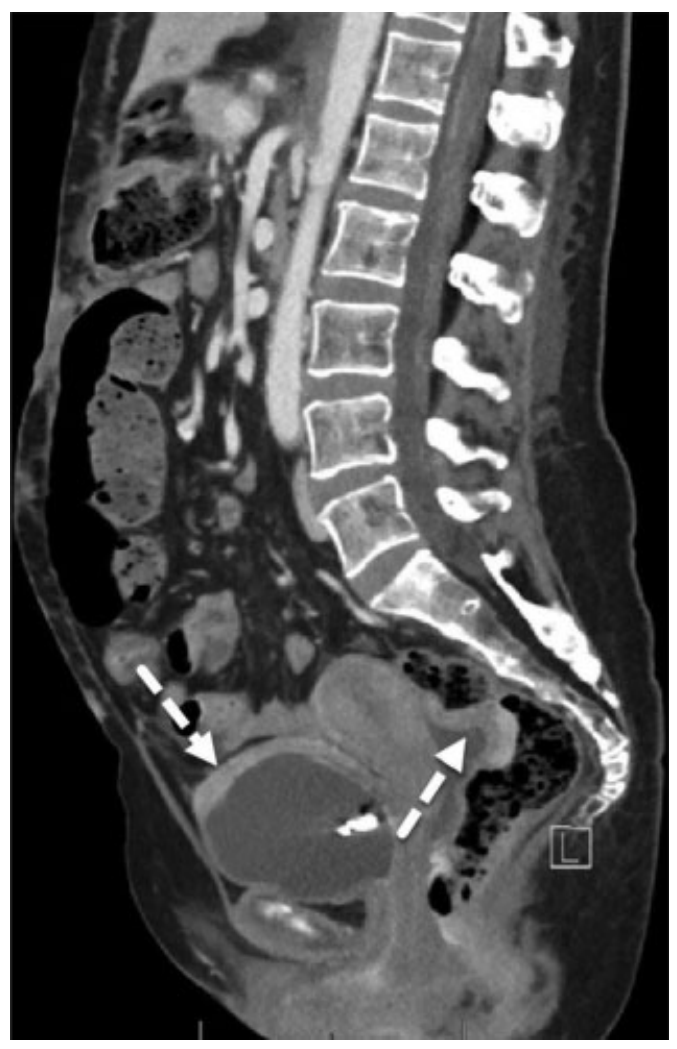

Fig. 20 A gastric adenocarcinoma patient with peritoneal implants (broken arrows) involving the rectouterine pouch (Blumer's shelf tumor) and along the dome of the urinary bladder. 

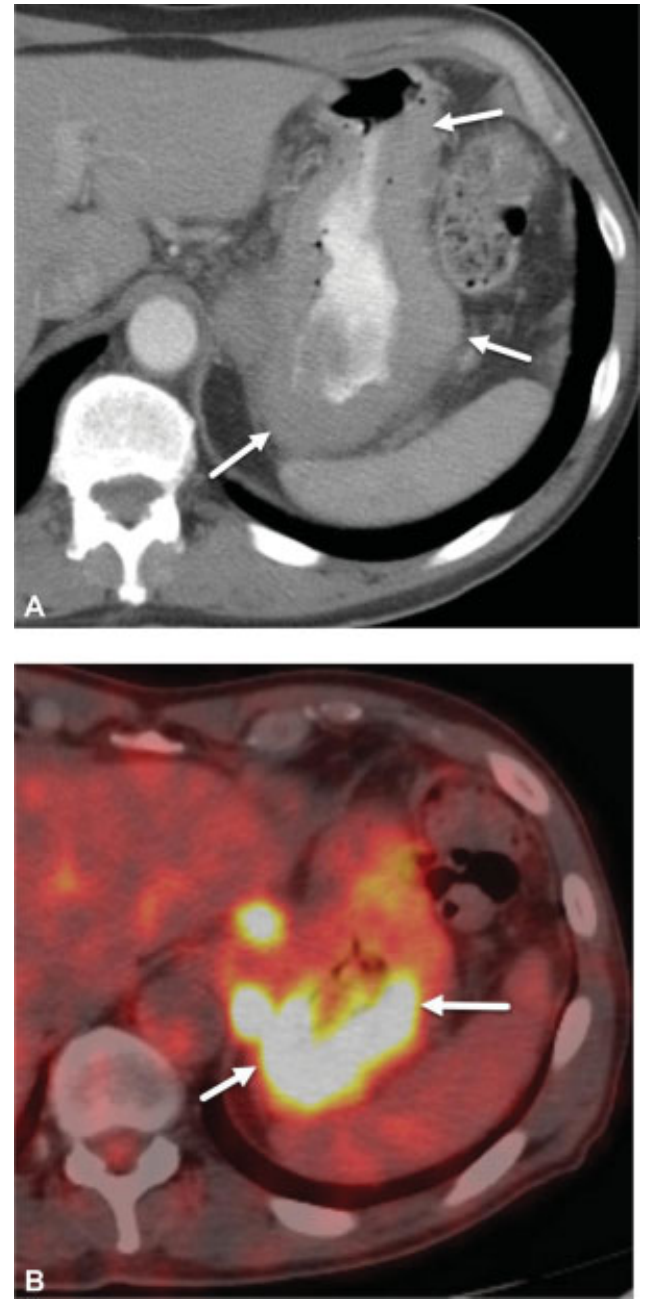

Fig. 21 Axial contrast enhanced computed tomography (CT) (A) and fluorodeoxyglucose (FDG) positron emission tomography (PET)-CT image (B) show diffuse thickening of the gastric wall (arrows), extending from the cardia to the antrum with elevated FDG uptake (SUVmax 22.5), mimicking infiltrative adenocarcinoma and linitis plastica. Biopsy revealed low grade marginal zone lymphoma of mucosa-associated lymphoid tissue (MALT) lymphoma.

Gastric lymphoma can mimic adenocarcinoma, and it may show polypoid, ulcerative or diffuse infiltrative macroscopic appearances. Infiltrative gastric lymphoma can cause extensive fold thickening and may mimic linitis plastica, but preservation of distensibility of the stomach and patency of its lumen can distinguish this entity from adenocarcinoma (-Fig. 21). Although GOO can occur in adenocarcinoma, it rarely occurs in gastric lymphoma. The presence of several polypoid lesions or ulcerating lesions may suggest the diagnosis of gastric lymphoma as opposed to adenocarcinoma. Absence of perigastric fat infiltration can also suggest the diagnosis of gastric lymphoma, especially in the setting of a large gastric mass (-Fig. 22). Although enlarged lymph nodes can occur in both entities, the presence of bulky lymph nodes or enlarged retroperitoneal lymph nodes, particularly below the level of the renal veins, is suggestive of gastric lymphoma. ${ }^{38}$

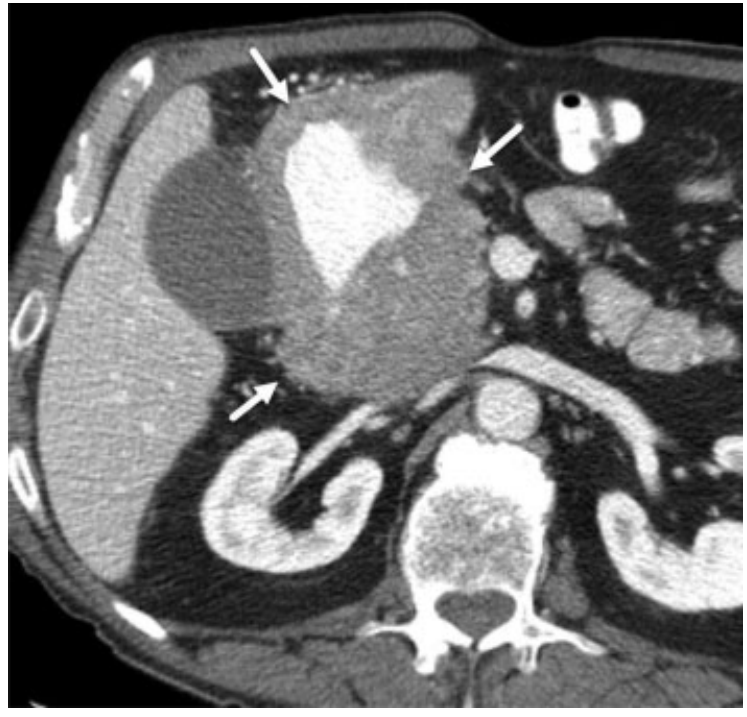

Fig. 22 Axial computed tomography image showing annular homogenous soft-tissue mass involving the gastric antrum (arrows). Despite extramural extension of the disease, there is no stranding of the adjacent fat planes, a feature suggestive of lymphoma. Biopsy revealed diffuse large B-cell lymphoma.
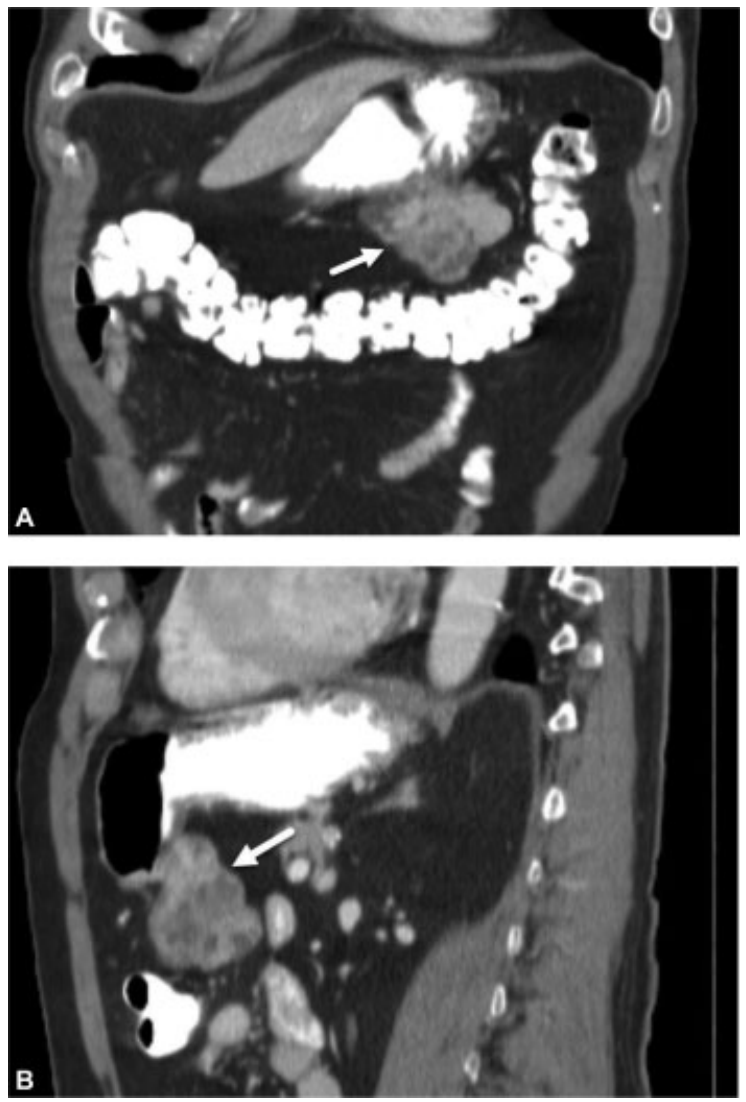

Fig. 23 Coronal (A) and sagittal reformatted computed tomography images (B) show a mass demonstrating exophytic growth along the greater curvature of the stomach (arrows). Gastrointestinal (GI) stromal tumor is the main differential of exophytic gastric masses, and it may have a homogenous or heterogenous texture on cross-sectional imaging. Final surgical pathology revealed a gastric gastrointestinal stromal tumors (GIST). 


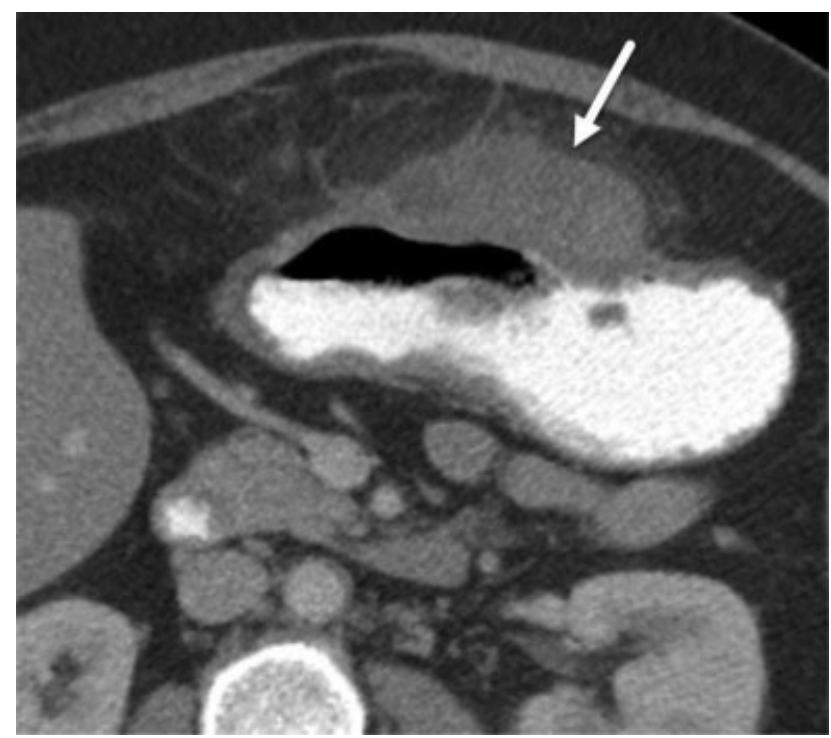

Fig. 24 Axial computed tomography image showing a homogenous exophytic gastric lesion, demonstrating exophytic growth pattern (arrow). Final surgical pathology revealed gastric gastrointestinal stromal tumor (GIST).

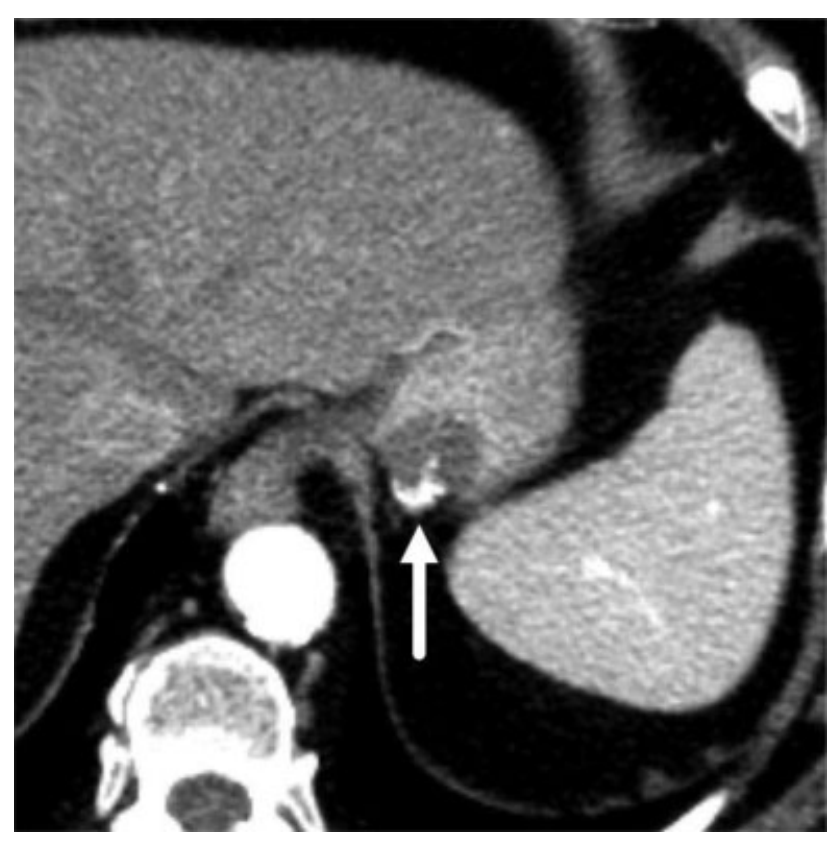

Fig. 25 Axial computed tomography image showing a small gastric mass, demonstrating intramural growth pattern within the collapsed gastric wall at the cardia, which also contains calcifications (arrow). Intramural growth pattern is uncommon in gastrointestinal stromal tumors (GIST). Calcifications and intraluminal growth are rarely seen in GISTs.

Gastric GISTs are characterized by exophytic growth pattern (-Figs. 23 and 24). Although an intramural growth

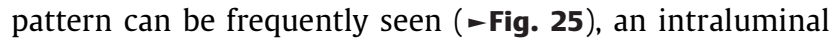
pattern of growth is uncommon. Most GISTs larger than $2 \mathrm{~cm}$ can have ulceration of the overlying mucosa, which can cause a bull's eye appearance on barium studies. Hemorrhagic or cystic degeneration can occur in GISTs (-Fig. 23), but calcifications are rare. Although liver metastases can occur in half of malignant GISTs, lymphatic dissemination and lymphadenopathy are uncommon. ${ }^{39}$

\section{Funding \\ None.}

\section{Conflict of Interest \\ None declared.}

\section{Acknowledgements}

The authors thank Dr. Rajesh Gothi for providing UGI barium examination images.

\section{References}

1 Bray F, Ferlay J, Soerjomataram I, Siegel RL, Torre LA, Jemal A. Global cancer statistics 2018: GLOBOCAN estimates of incidence and mortality worldwide for 36 cancers in 185 countries. CA Cancer J Clin 2018;68(06):394-424

2 Collaborators GBDSC GBD 2017 Stomach Cancer Collaborators. The global, regional, and national burden of stomach cancer in 195 countries, 1990-2017: a systematic analysis for the Global Burden of Disease study 2017. Lancet Gastroenterol Hepatol 2020;5(01):42-54

3 Leung WK, Wu MS, Kakugawa Y, et al; Asia Pacific Working Group on Gastric Cancer. Screening for gastric cancer in Asia: current evidence and practice. Lancet Oncol 2008;9(03):279-287

4 Vikram R, Balachandran A, Bhosale PR, Tamm EP, Marcal LP, Charnsangavej C. Pancreas: peritoneal reflections, ligamentous connections, and pathways of disease spread. Radiographics 2009;29(02):e34

5 Young JJ, Pahwa A, Patel M, et al. Ligaments and lymphatic pathways in gastric adenocarcinoma. Radiographics 2019;39 (03):668-689

6 Ajani JA, Curley SA, Janjan NA, Lynch PM. Gastrointestinal Cancer. New York, NY: Springer; 2005

7 Lauren P. The two histological main types of gastric carcinoma: diffuse and so-called intestinal-type carcinoma. An attempt at a histo-clinical classification. Acta Pathol Microbiol Scand 1965; 64:31-49

8 Hamilton S, Aaltonen L. World Health Organization Classification of Tumours. Pathology and Genetics of Tumours of the Digestive System Lyon: IARC Press; 2000

9 Dicken BJ, Bigam DL, Cass C, Mackey JR, Joy AA, Hamilton SM. Gastric adenocarcinoma: review and considerations for future directions. Ann Surg 2005;241(01):27-39

10 Fenogilo-Preiser C, Carneiro F, Correa P, et al. Gastric carcinoma. In: Hamilton SAL, ed. Pathology and Genetics. Tumors of the Digestive System Vol. 1;Lyon: Lyon Press; 2000:37-52

11 Brooks-Wilson AR, Kaurah P, Suriano G, et al. Germline E-cadherin mutations in hereditary diffuse gastric cancer: assessment of 42 new families and review of genetic screening criteria. J Med Genet 2004;41(07):508-517

12 Borrman R. Geschwulste des Magens und Duodenums. In: Henke F, Lubarsch O, eds. Handbuch der speziellen pathologischen Anatomie und Histologie. Berlin: Springer; 1926

13 American Joint Committee on Cancer. Stomach. In: Amin MB Edge SB, Greene F, Byrd DR, Brookland R, Washington MK, eds. AJCC Cancer Staging Manual. 8th ed. New York: Springer-Verlag; 2018

14 Adachi Y, Shiraishi N, Suematsu T, Shiromizu A, Yamaguchi K Kitano S. Most important lymph node information in gastric cancer: multivariate prognostic study. Ann Surg Oncol 2000;7 (07):503-507 
15 Roder JD, Böttcher K, Busch R, Wittekind C, Hermanek P, Siewert JRGerman Gastric Cancer Study Group. Classification of regional lymph node metastasis from gastric carcinoma. Cancer 1998;82 (04):621-631

16 Siewert JR, Böttcher K, Stein HJ, Roder JD. Relevant prognostic factors in gastric cancer: ten-year results of the German Gastric Cancer Study. Ann Surg 1998;228(04):449-461

17 Yokota T, Kunii Y, Teshima S, et al. Significant prognostic factors in patients with early gastric cancer. Int Surg 2000;85(04):286-290

18 Japanese Gastric Cancer Association. Japanese Classification of Gastric Carcinoma - 2nd English Edition -. Gastric Cancer 1998;1(01):10-24

19 Sobin L, Wittekind C. UICC TNM Classification of Malignant Tumours. 6th ed. New York: Wiley; 2002

20 Brennan MF. Current status of surgery for gastric cancer: a review. Gastric Cancer 2005;8(02):64-70

21 Takao M, Fukuda T, Iwanaga S, Hayashi K, Kusano H, Okudaira S. Gastric cancer: evaluation of triphasic spiral CT and radiologicpathologic correlation.J Comput Assist Tomogr 1998;22(02):288-294

22 Smyth EC, Verheij M, Allum W, Cunningham D, Cervantes A, Arnold DESMO Guidelines Committee. Gastric cancer: ESMO Clinical Practice Guidelines for diagnosis, treatment and followup. Ann Oncol 2016;27(Suppl 5):v38-v49

23 Hamashima CSystematic Review Group and Guideline Development Group for Gastric Cancer Screening Guidelines. Update version of the Japanese Guidelines for Gastric Cancer Screening. Jpn J Clin Oncol 2018;48(07):673-683

24 Karpeh MS Jr, Brennan MF. Gastric carcinoma. Ann Surg Oncol 1998;5(07):650-656

25 Bhandari S, Shim CS, Kim JH, et al. Usefulness of three-dimensional, multidetector row CT (virtual gastroscopy and multiplanar reconstruction) in the evaluation of gastric cancer: a comparison with conventional endoscopy, EUS, and histopathology. Gastrointest Endosc 2004;59(06):619-626

26 Kelly S, Harris KM, Berry E, et al. A systematic review of the staging performance of endoscopic ultrasound in gastro-oesophageal carcinoma. Gut 2001;49(04):534-539

27 Hargunani R, Maclachlan J, Kaniyur S, Power N, Pereira SP, Malhotra A. Cross-sectional imaging of gastric neoplasia. Clin Radiol 2009;64(04):420-429
28 Chen CY, Hsu JS, Wu DC, et al. Gastric cancer: preoperative local staging with 3D multi-detector row CT-correlation with surgical and histopathologic results. Radiology 2007;242(02):472-482

29 Japanese Gastric Cancer Association. Japanese classification of gastric carcinoma: 3rd English edition. Gastric Cancer 2011;14 (02):101-112

30 Lim JS, Yun MJ, Kim MJ, et al. CT and PET in stomach cancer: preoperative staging and monitoring of response to therapy. Radiographics 2006;26(01):143-156

31 Stahl A, Ott K, Weber WA, et al. FDG PET imaging of locally advanced gastric carcinomas: correlation with endoscopic and histopathological findings. Eur J Nucl Med Mol Imaging 2003;30(02):288-295

32 Yoshioka T, Yamaguchi K, Kubota K, et al. Evaluation of 18F-FDG PET in patients with advanced, metastatic, or recurrent gastric cancer. J Nucl Med 2003;44(05):690-699

33 Mönig SP, Zirbes TK, Schröder W, et al. Staging of gastric cancer: correlation of lymph node size and metastatic infiltration. AJR Am J Roentgenol 1999;173(02):365-367

34 Sohn KM, Lee JM, Lee SY, Ahn BY, Park SM, Kim KM. Comparing MR imaging and CT in the staging of gastric carcinoma. AJR Am J Roentgenol 2000;174(06):1551-1557

35 Harisinghani MG, Saini S, Weissleder R, et al. MR lymphangiography using ultrasmall superparamagnetic iron oxide in patients with primary abdominal and pelvic malignancies: radiographic-pathologic correlation. AJR Am J Roentgenol 1999;172(05):1347-1351

36 Kim AY, Han JK, Seong CK, Kim TK, Choi BI. MRI in staging advanced gastric cancer: is it useful compared with spiral CT? J Comput Assist Tomogr 2000;24(03):389-394

37 Mawlawi O, Podoloff DA, Kohlmyer S, et al; National Electrical Manufacturers Association. Performance characteristics of a newly developed PET/CT scanner using NEMA standards in 2D and 3D modes. J Nucl Med 2004;45(10):1734-1742

38 Ghai S, Pattison J, Ghai S, O'Malley ME, Khalili K, Stephens M. Primary gastrointestinal lymphoma: spectrum of imaging findings with pathologic correlation. Radiographics 2007;27(05): 1371-1388

39 Kang HC, Menias CO, Gaballah AH, et al. Beyond the GIST: mesenchymal tumors of the stomach. Radiographics 2013;33 (06):1673-1690 